\title{
Multistability in nonlinear left-handed transmission lines
}

\author{
David A. Powell, ${ }^{\text {a) }}$ llya V. Shadrivov, and Yuri S. Kivshar \\ Nonlinear Physics Center, Research School of Physical Sciences and Engineering, \\ Australian National University, Canberra ACT 0200, Australia
}

(Received 30 April 2008; accepted 15 June 2008; published online 2 July 2008)

Employing a nonlinear left-handed transmission line as a model system, we demonstrate experimentally the multistability phenomena predicted theoretically for microstructured left-handed metamaterials with a nonlinear response. We show that the bistability is associated with the period doubling, which at higher power may result in chaotic dynamics of the transmission line. (C) 2008 American Institute of Physics. [DOI: 10.1063/1.2955531]

Left-handed metamaterials are artificial structures having negative effective electric and magnetic parameters over some frequency range. ${ }^{1}$ These structures can be characterized by an effective negative refractive index, and they support the propagation of backward waves, where the wave fronts propagate in the direction opposite to the energy flow defined by the Poynting vector. ${ }^{2}$ This important property enables several other interesting physical phenomena, particularly subwavelength imaging. ${ }^{3}$ It is also of significant interest when combined with nonlinearity, since it enables frequency tuning, switching between opaque and transparent states, and efficient second-harmonic reflection. ${ }^{4}$

Left-handed transmission lines created by arrays of identical lumped capacitors in series and shunt inductors also support the propagation of backward waves. Thus such transmission lines provide more compact and easily studied analog of left-handed metamaterials, and when constructed in three-dimensional arrays they can also form true metamaterials. ${ }^{5}$ Such structures are sometimes termed composite right-handed/left-handed transmission lines, since the unavoidable series inductive and shunt capacitance results in a right-handed (i.e., forward-wave) passband.

Nonlinear left-handed transmission lines have been studied for potential device applications, and more recently for their insight into the physics of left-handed metamaterials. They have been shown to exhibit mismatched harmonic generation, ${ }^{6}$ parametric generation and amplification, ${ }^{7}$ and envelope solitons. ${ }^{8}$ In this letter we study the properties of a nonlinear left-handed transmission line and demonstrate a multistable response in its left-handed passband, similar to the multistability predicted theoretically for nonlinear metamaterials.

We study a microstrip transmission line constructed on $1.6 \mathrm{~mm}$ copper-clad FR4. The track sections are $3.4 \mathrm{~mm}$ wide and $10 \mathrm{~mm}$ long, with $0.75 \mathrm{~mm}$ gaps to allow the insertion of lumped capacitance and holes to contain the shunt inductors. The lumped nonlinear capacitance is provided by SMV1405 hyperabrupt junction varactor diodes, and the inductance by $18 \mathrm{nH}$ chip inductors. The equivalent circuit of each (asymmetric) unit cell is shown in Fig. 1(a). This is a much simpler geometry than that presented in Ref. 6 since it has only a single diode per unit cell and unlike the geometry presented in Ref. 9 the line is not coupled to external resonant elements.

\footnotetext{
a)Electronic mail: david.a.powell@anu.edu.au.
}

The geometry and lumped element values were chosen to ensure a significant stop band between the right-handed and left-handed passbands so that all the regimes of wave propagation in the composite right-/left-handed structure are present. The line was 20 periods long to ensure that end effects did not dominate the response. As shown in Fig. 1(b), the left-handed passband extends from 400 to $900 \mathrm{MHz}$, with the right-handed band starting at approximately 1.5 GHz. The structure of the waves in the transmission line was verified by scanning the field distribution using a probe attached to a linear translation stage and vector network analyzer. The resultant phase distributions are shown in Fig. 2, where it can be seen that in the right-handed band the phase decreases further away from the source, whereas in the lefthanded band it increases.

All measurements were made in a $50 \Omega$ system; however, the inherent dispersion of the left-handed transmission line means that the characteristic impedance of the line will vary with frequency, and this will result in reflections from the connectors. For this reason the phase plots show some variation from linearity near the connectors. The nonsmooth character of the curves is due to the internal structure of the electric field distribution on the scale of the transmission line unit cell.

In order to measure the nonlinear transmission response, a signal generator was applied to the input port and the output port was connected to a spectrum analyzer. The spectrum

(a)
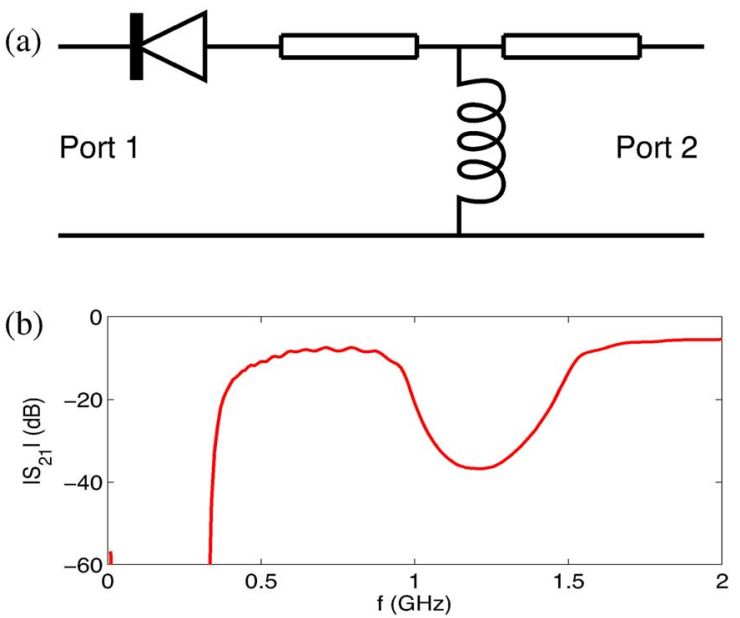

FIG. 1. (Color online) Unit cell (a) and linear frequency response (b) of the nonlinear left-handed transmission line. 


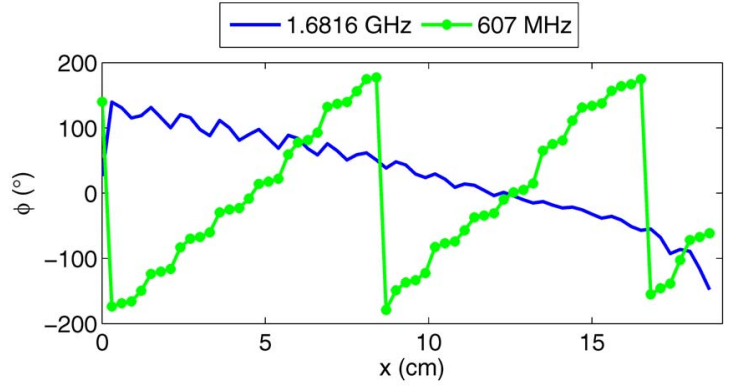

FIG. 2. (Color online) Spatial phase distribution at $1.6816 \mathrm{GHz}$ in the righthanded band and $607 \mathrm{MHz}$ in the left-handed band.

from $10 \mathrm{MHz}$ to $2 \mathrm{GHz}$ was captured in 501 points with a resolution bandwidth of $3 \mathrm{MHz}$ and video bandwidth of $30 \mathrm{kHz}$. A sinusoidal signal of frequency $600 \mathrm{MHz}$ was applied, which falls within the left-handed passband. The input power was swept in steps of $0.1 \mathrm{~dB}$ from 1 to $8 \mathrm{dBm}$. Below $1 \mathrm{dBm}$ the system showed an essentially linear relationship between input and output power as well as the expected harmonic generation.

Due to the asymmetry of the diodes (which are all identically oriented), the transmission responses in different directions are not identical in the nonlinear regime. Figure 3 shows the magnitude of the fundamental frequency as a function of input power applied to port 1 [as defined in Fig. 1(a)]. It can be seen that there are two regions of multistability within this power range.

Figure 4 shows the measured spectrum for an input power of $4.5 \mathrm{dBm}$ swept in the forward and reverse directions. The forward sweep spectrum is representative of the whole branch from the lowest measured power up to $4.9 \mathrm{dBm}$. A strong fundamental frequency can be seen, as well as strong second- and third-harmonic components. We note that the second harmonic falls within the stop band; however, at this frequency, the transmission in the stop band is only $25 \mathrm{~dB}$ lower than in the passband, thus accounting for the relatively strong second-harmonic component.

The spectrum in the reverse sweep is representative of the lower branch of the hysteresis curve (see Fig. 3) extending from 3.1 to $6.5 \mathrm{dBm}$ (i.e., up to but not including the upper branch of the second bistable region). The subharmonic component corresponding to a period doubling bifurcation is clearly visible in this regime; however, it falls within the lowest order cutoff band and is strongly attenuated. Other fractional frequency components are much stronger, particular $3 / 2 f_{0}$, which falls within the left-handed

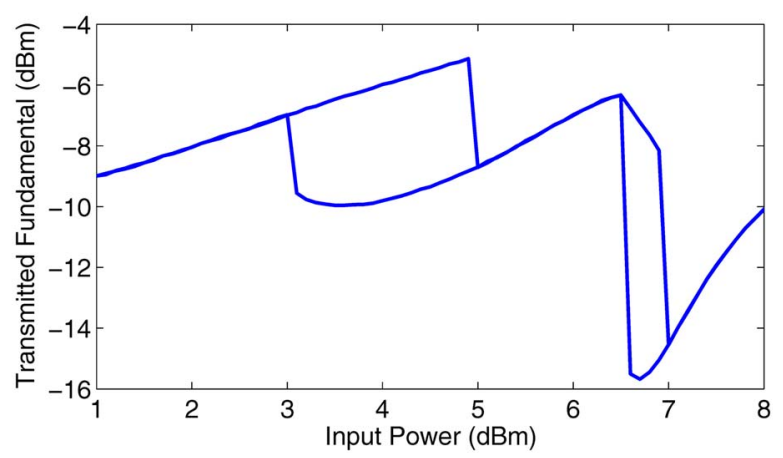

FIG. 3. (Color online) Output at fundamental frequency with swept input power at $600 \mathrm{MHz}$, showing two regions of bistability. (a) Forward Sweep

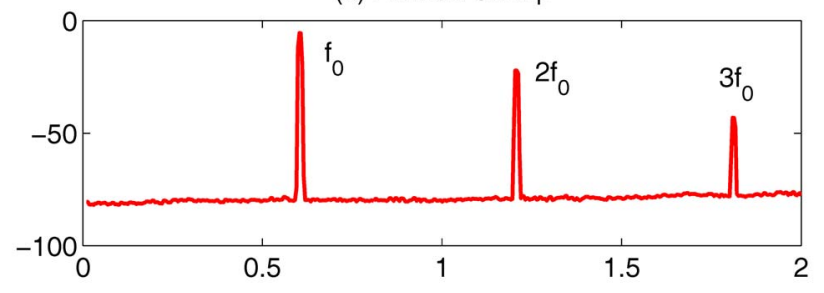

(b) Reverse Sweep

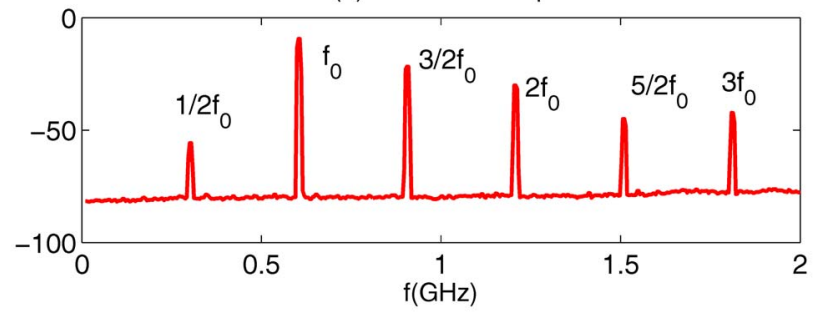

FIG. 4. (Color online) Output spectrum for input power of $4.5 \mathrm{dBm}$ for forward (a) and reverse (b) sweeps. The reverse branch shows fractional frequency components corresponding to period doubling.

transmission band. The effect of multistability on our nonlinear transmission line has a different physical origin to that in the the nonlinear left-handed metamaterials. While in metamaterials we observe a nonlinear shift of the resonance response, in transmission lines the transmission bands do not change, and the existence of several dynamic states for the same input power is related to the period doubling. The period doubling clearly manifests itself in subharmonic generation for the reverse sweep shown in Fig. 4. It is well known that period doubling is one of the possible routes towards chaotic behavior in a system. Indeed, for higher powers of the signal wave, the spectrum of the transmitted wave has a quasicontinuous structure (see Fig. 5), which indicates chaotic behavior (see, e.g., Ref. 10).

To gain a broader picture of the bistable behavior, a map of bistable regions in frequency-power space was produced, as shown in Fig. 6. This was done by performing a bidirectional power sweep for frequencies in between 500 and

(a) Forward Sweep

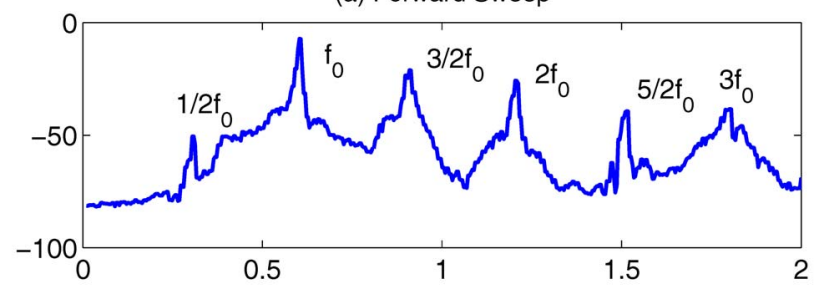

(b) Reverse Sweep

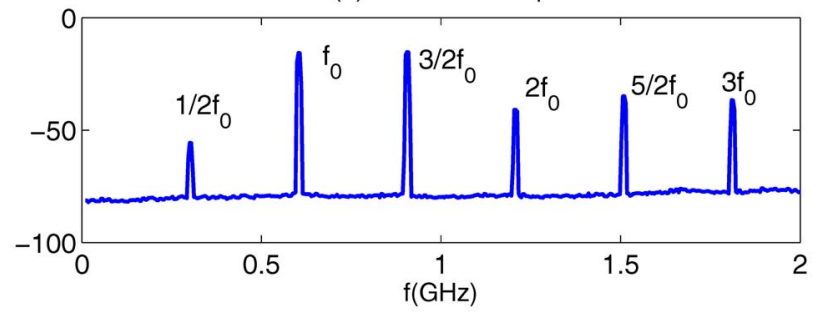

FIG. 5. (Color online) Output spectrum for input power of $6.7 \mathrm{dBm}$ for forward (a) and reverse (b) sweeps. The forward sweep shows a quasicontinuous spectrum indicative of chaos, and the reverse sweep shows period doubling. 


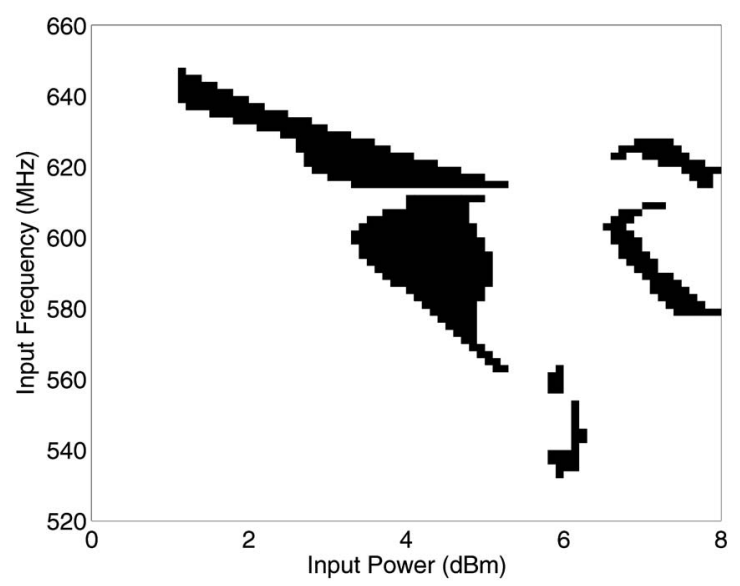

FIG. 6. Regions of bistability as a function of input frequency and power of a sinusoidal signal applied to port 1 .

$700 \mathrm{MHz}$ in $2 \mathrm{MHz}$ steps (the figure has been limited to those regions where a bistable response was found). A threshold of $0.04 \mathrm{~dB}$ difference in forward and reverse power sweeps was used to eliminate the effect of measurement noise. This shows that several distinct regions of bistability exist, and their frequencies suggest that the mechanism is intimately related to the fundamental frequency being in the left-handed propagation band. The range of frequencies also coincides with $2 f_{0}$ lying in the stop band, $f_{0} / 2$ lying within the low-pass stop band, and $3 / 2 f_{0}$ lying within the lefthanded passband, although it is not clear how each of these factors contributes to the observed behavior.

For comparison purposes the fundamental response of the system was also analyzed when the input is applied to port 2 (not shown). The critical points of bistability are significantly different from those in the input at port 1 . This is due to the asymmetry in the properties of the diodes relative to the source, which along with the nonlinearity produces a nonreciprocal response of the structure.

These multistability results are due to a different mechanism than that previously reported by the authors for a var- actor loaded split ring resonator (SRR) ${ }^{11}$ For the SRR the multistability was linked to the accumulation of charge across the reverse-biased varactor diode, which resulted in the shift of the resonance band of the resonators. In this case there is negligible frequency shift of the band gaps; however, several dynamic states can coexist in the nonlinear structure at an appropriate power level.

In conclusion, we have experimentally studied the multistability phenomenon which occurs in left-handed metamaterials with a nonlinear response. We have employed a nonlinear left-handed transmission line as a model system, and have demonstrated a multistable response in its left-handed passband, similar to the multistability predicted theoretically for nonlinear metamaterials. We have shown that the bistability is associated with period doubling, which may result in chaotic dynamics at higher power.

The authors thank Dr. Thomas Stemler for helpful discussions and acknowledge support from the Australian Research Council through the Discovery Project.

${ }^{1}$ D. Smith, W. Padilla, D. Vier, S. Nemat-Nasser, and S. Schultz, Phys. Rev. Lett. 84, 4184 (2000).

${ }^{2}$ V. Veselago, Sov. Phys. Usp. 10, 509 (1968).

${ }^{3}$ J. B. Pendry, Phys. Rev. Lett. 85, 3966 (2000).

${ }^{4}$ I. V. Shadrivov and Yu. S. Kivshar, in Physics of Negative Refraction and Negative Index Materials, Springer Series in Materials Science Vol. 98, edited by C. M. Krowne and Y. Zhang (Springer, New York, 2007).

${ }^{5}$ A. K. Iyer and G. V. Eleftheriades, IEEE Trans. Antennas Propag. 55, 2746 (2007).

${ }^{6}$ A. B. Kozyrev, H. Kim, A. Karbassi, and D. W. van der Weide, Appl. Phys. Lett. 87, 121109 (2005).

${ }^{7}$ A. Kozyrev, H. Kim, and D. van der Weide, Appl. Phys. Lett. 88, 264101 (2006).

${ }^{8}$ A. B. Kozyrev and D. W. van der Weide, Appl. Phys. Lett. 91, 254111 (2007).

${ }^{9}$ I. Gil, J. Garcia-Garcia, J. Bonache, F. Martin, M. Sorolla, and R. Marques, Electron. Lett. 40, 1347 (2004).

${ }^{10}$ H. G. Schuster and W. Just, Deterministic Chaos. An Introduction (WileyVCH, Weinheim, 2004).

${ }^{11}$ D. A. Powell, I. V. Shadrivov, Yu. S. Kivshar, and M. V. Gorkunov, Appl. Phys. Lett. 91, 144107 (2007). 